\title{
A flexible and transparent ceramic nanobelt network for soft electronics
}

\author{
Siya Huang ${ }^{1}$, Hui $\mathrm{Wu}^{1}$, Ming Zhou ${ }^{2}$, Chunsong Zhao ${ }^{1}$, Zongfu Yu${ }^{2}$, Zhichao Ruan ${ }^{3}$ and Wei Pan ${ }^{1}$ \\ In the near future, scientists and researchers hope to use semiconducting materials in various human-friendly electronic \\ devices such as skin-like sensors and interactive electronics, which require them to function properly while being bent, \\ stretched or twisted. In this work, we managed to achieve excellent mechanical flexibility in conventionally fragile ceramics with \\ a design of nanobelt network. We engineered inorganic oxides into ultralong and continuous nanobelts via an extremely simple \\ and scalable electrospinning process. The as-synthesized $\mathrm{SnO}_{2}$ nanobelts possess ultrahigh aspect ratios $\left(>10^{5}\right)$ and well- \\ defined rectangular cross-section, which exhibit outstanding mechanical flexibility under a bending radius down to $1 \mathrm{~mm}$ and \\ show no obvious electrical degradation after 1000 cycles of bending to a radius of $2 \mathrm{~mm}$. Moreover, the free-standing nanobelt \\ network demonstrates superior optoelectronic properties as well as high optical transparency ( $>80 \%$ transmittance at $550 \mathrm{~nm}$ ), \\ which enables us to construct conformable and 'invisible' UV photodetectors on multiple flexible or curved substrates, including \\ plastics, paper, textiles and curved/bio-surfaces. These results strongly indicate the great compatibility and potential of \\ inorganic nanobelt networks as flexible and transparent functional electronics.
}

NPG Asia Materials (2014) 6, e86; doi:10.1038/am.2013.83; published online 14 February 2014

Keywords: electrospinning; flexible electronics; nanobelts; tin oxides

\section{INTRODUCTION}

Inorganic semiconductors have been employed as key components in a wide range of areas, such as field effect transistors, ${ }^{1}$ light emitting diodes, ${ }^{2-4}$ sensors ${ }^{5,6}$ and piezoelectric elements. ${ }^{7}$ However, their applications in flexible electronics are severely confined due to the brittleness. In this regard, intensive studies have been conducted on exploiting advanced fabrication techniques and materials suitable for soft electronics. Materials with improved mechanical flexibility have been reported, such as two-dimensional (2D) topological insulators ${ }^{8}$ and graphene films, ${ }^{9}$ one-dimensional (1D) nanostructures ${ }^{10}$ and carbon nanotubes ${ }^{11}$ and zero-dimensional (OD) chalcogenide nanocrystals. ${ }^{12,13}$ Rogers' group has pioneered epidermal electronics with controlled geometrical and spatial configurations in design of flexible electronics. ${ }^{14-16}$ Hosono and co-workers demonstrated a class of amorphous oxide semiconductor films as transparent flexible transistors. ${ }^{17}$ Despite the enormous progress achieved so far, it still remains a great challenge to produce inorganic semiconductors with extreme deformability in a scalable way. Moreover, efficient integration of these materials with improved mechanical flexibility into flexible functional devices, especially on substrates with different textures and complex curvilinear surfaces, is far from well developed.

$\mathrm{SnO}_{2}$, as an important wide-bandgap semiconductor, has been widely explored as gas sensors ${ }^{18,19}$ and solar-blind UV detectors, ${ }^{20,21}$ whereas further applications in flexible electronics have been rarely reported due to its poor mechanical properties. One solution is to synthesize and incorporate micro/nano-structures, such as wires, ribbons and bars, which can tolerate mechanical deformation to a certain extent. ${ }^{22}$ However, some limitations, such as non-uniformity with low aspect ratios, unstable cross-junctions with high resistance and sophisticated assembly setups, are still standing in the way of achieving sufficient and durable flexibility in practical applications. Accordingly, new strategies need to be developed, which should meet the requirements of controlled synthesis of highly uniform nanostructures with tailored geometry, reliable interconnection with low junction resistance and facile integration with multiple flexible or curved substrates.

\section{EXPERIMENTAL PROCEDURES}

Sample preparation and characterization

$\mathrm{SnO}_{2}$ nanobelts were synthesized via a combination of sol-gel-based electrospinning and subsequent heat treatment. The precursor solution was prepared by dissolving $\mathrm{SnCl}_{4} \cdot 5 \mathrm{H}_{2} \mathrm{O}(0.1 \mathrm{~g})$ in pure ethanol $(3.8 \mathrm{ml})$, and then mixed with $5.0 \mathrm{wt} . \%$ polyvinyl butyral. The mixture solution was stirred till it turned into a clear sol, and then delivered into a syringe with a stainless steel needle. A voltage of $12 \mathrm{kV}$ was applied to the sol with a high-voltage power supply. Grounded aluminum foil was placed below the syringe at a distance of $15 \mathrm{~cm}$ to collect nonwoven fiber mats. After a period of deposition time, the fiber mats were peeled off as a free-standing film and calcined in air at $500{ }^{\circ} \mathrm{C}$ for $2 \mathrm{~h}$

\footnotetext{
${ }^{1}$ State Key Laboratory of New Ceramics and Fine Processing, School of Materials Science \& Engineering, Tsinghua University, Beijing, PR China; ${ }^{2}$ Department of Electrical and Computer Engineering, University of Wisconsin, Madison, WI, USA and ${ }^{3}$ Department of Physics, Zhejiang University, Hangzhou, PR China

Correspondence: Professor H Wu or Professor W Pan, State Key Laboratory of New Ceramics and Fine Processing, School of Materials Science \& Engineering, Tsinghua University, Beijing 100084, PR China.

E-mail: huiwu@tsinghua.edu.cn or panw@tsinghua.edu.cn

Received 2 November 2013; revised 13 November 2013; accepted 17 November 2013
} 
at a heating rate of $5^{\circ} \mathrm{Cmin}^{-1}$ to form $\mathrm{SnO}_{2}$ nanobelt networks. $\mathrm{SnO}_{2}$ films were prepared on $100-\mu \mathrm{m}$-thick polyethylene terephthalate (PET) substrates using a.c. magnetosputtering (LJ-SP103C, LJ-UHV Technology Co. Ltd., Taiwan) with a power of $150 \mathrm{~W}$ at a pressure of $20 \mathrm{mtorr}$. The film thickness was $\sim 100 \mathrm{~nm}$ based on calculation. The samples were characterized with field emission scanning electron microscopy (SEM, JSM-7001F, JEOL Ltd., Tokyo, Japan), transmission electron microscopy (TEM, JEM-2010, JEOL Ltd.) and X-ray diffraction (XRD, D/max-2500, Rigaku Ltd., Tokyo, Japan).

\section{Optoelectronic and optical tests}

Time-dependent on/off photoresponse was conducted on Keithley 4200-SCS measurement system. Samples were located on a probe station in a shielded box. The current was monitored while UV light (Philips TUV $8 \mathrm{~W}, 254 \mathrm{~nm}$ ) was turned on and off in a time interval of $2 \mathrm{~min}$. The specular transmittance was measured by using a UV-visible spectrophotometer (TU-1810PC, Purkinje General Corp., Beijing, China). The samples were transferred onto quartz substrates. The transmittance of a plain quartz substrate was set as baseline.

\section{RESULTS AND DISCUSSION}

Synthesis and characterization of $\mathrm{SnO}_{2}$ nanobelts

In this study, we managed to achieve extraordinary flexibility in conventionally fragile $\mathrm{SnO}_{2}$ by fabricating a network of intertwined $\mathrm{SnO}_{2}$ nanobelts. Electrospinning, as one of the most efficient methods to produce ultralong $1 \mathrm{D}$ nanostructures with low cost and high scalability, ${ }^{23,24}$ was employed to synthesize the $\mathrm{SnO}_{2}$ nanobelts. Particularly, the size, morphology, geometry and chemical composition of electrospun nanostructures can be easily tuned by adjusting the precursor constituents and operating parameters (e.g. applied voltage, working distance and the feeding rate of solution). ${ }^{23}$ Accordingly, we were able to produce continuous nanofibers with a tailored ribbon-like shape. Ribbon-like nanostructures with rectangular cross-section could form during electrospinning through rapid solvent evaporation, which may cause a dry skin on the surface of liquid jet and lead to subsequent nanofiber collapse. ${ }^{23,25,26}$ As shown in Supplementary Figure S1, the as-electrospun composite fibermats consist of continuous nanobelts with smooth surfaces. Figure 1a shows the SEM images of a typical electrospun web after calcination. We can see that the as-obtained products preserve the highly uniform belt-like structure with an average width of $\sim 250 \mathrm{~nm}$ and thickness of $\sim 35 \mathrm{~nm}$ (Supplementary Figure S2). XRD pattern (Supplementary Figure S3) shows that all the diffraction peaks can be well indexed to rutile $\mathrm{SnO}_{2}$ (JCPDS card no. 77-0449). Further analysis with TEM, selected area electron diffraction (SAED) and high-resolution TEM (HRTEM) confirm that the as-synthesized nanobelts are polycrystalline $\mathrm{SnO}_{2}$ with grains $<10 \mathrm{~nm}$ (Supplementary Figure S4).
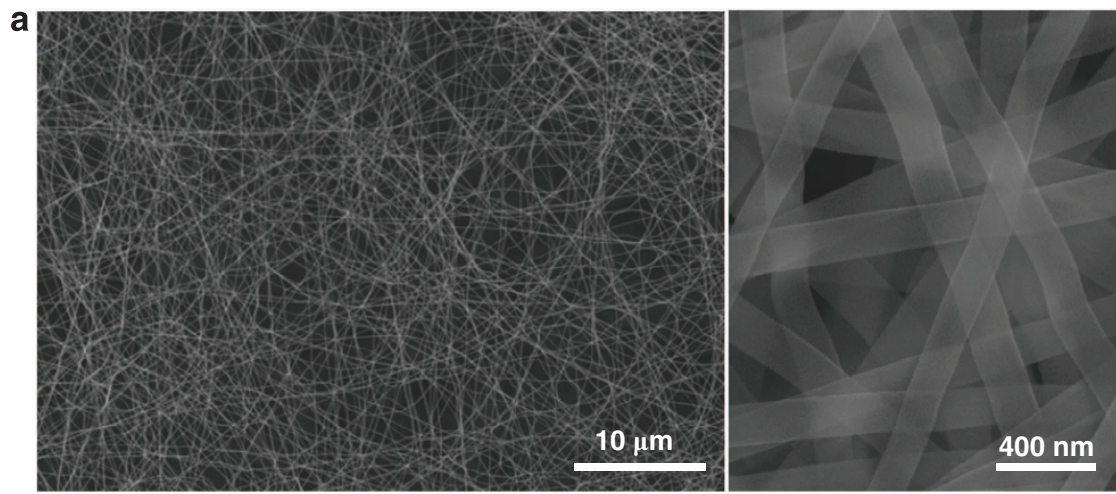

b
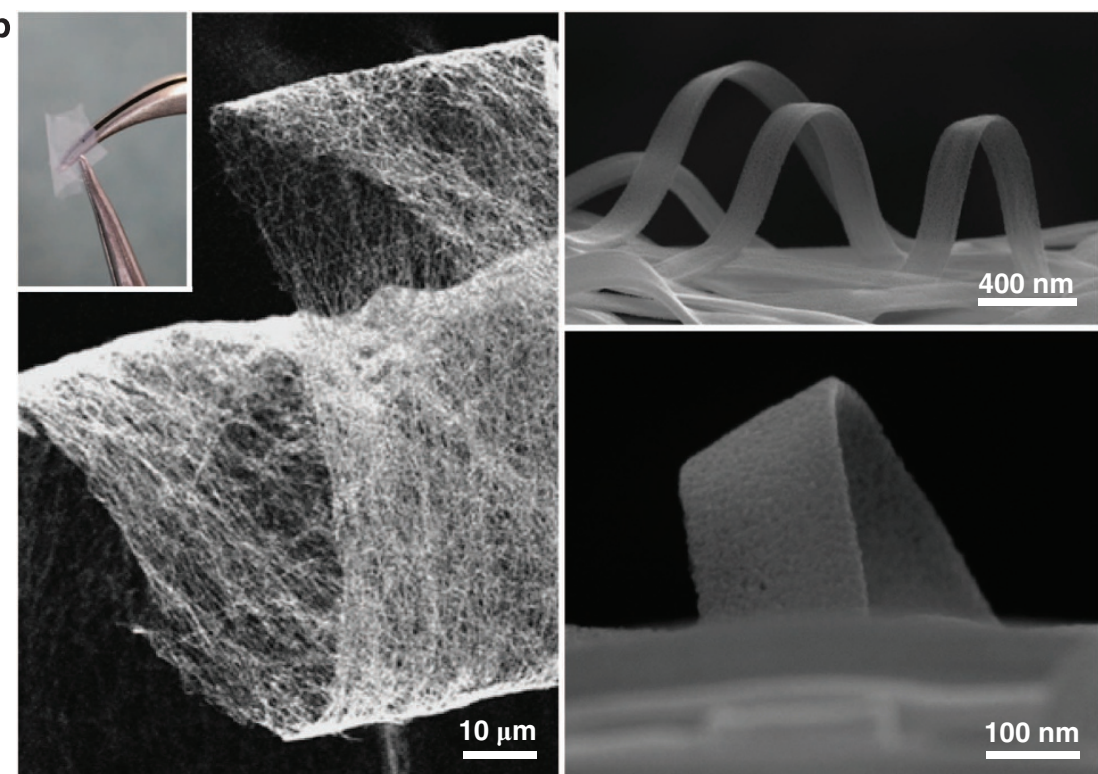

Figure 1 Morphology and microstructure of the $\mathrm{SnO}_{2}$ nanobelt network. (a) Top-view $\mathrm{SEM}$ images of the $\mathrm{SnO}_{2}$ nanobelt network at low and high magnifications. (b) SEM images of a folded free-standing $\mathrm{SnO}_{2}$ nanobelt network at different magnifications. Inset: a digital photo of the folded free-standing $\mathrm{SnO}_{2}$ nanobelt network. 
Mechanical flexibility of $\mathrm{SnO}_{2}$ nanobelt network

The electropun nanobelt is continuous with length $>1 \mathrm{~cm}$ (Supplementary Figure S5), which results in an ultrahigh aspect ratio above $10^{5}$. Such long nanobelts are likely to form a uniform and closely intertwined network over a large area without any binder. As shown in the inset of Figure 1b, the free-standing $\mathrm{SnO}_{2}$ nanobelt web (area $\sim 1 \mathrm{~cm} \times 2 \mathrm{~cm}$ ) can be easily lifted and folded with forceps. The left SEM image in Figure 1b clearly shows that the $\mathrm{SnO}_{2}$ nanobelt web can be bent down to a radius $<10 \mu \mathrm{m}$ without any fracture. Further observations on a single nanobelt (right panel of Figure $1 \mathrm{~b}$ ) demonstrate that each nanobelt can tolerate extreme deformations with $r$ $<200 \mathrm{~nm}$ (corresponding to a strain $>10 \%$ ), which endows the nanobelt network with superior mechanical flexibility over traditional materials.

Owing to the large aspect ratio, nanoscale ribbon-like geometry and a well-interconnected web configuration, our $\mathrm{SnO}_{2}$ nanobelt network presents remarkable mechanical flexibility and durability. To examine its mechanical and optoelectronic properties, we transferred a $1 \mathrm{~cm} \times 1 \mathrm{~cm} \mathrm{SnO}_{2}$ nanobelt film onto a $100-\mu \mathrm{m}$-thick PET substrate. This whole process can be finished manually under visual observation, which strongly demonstrates its operability and attractive cost-efficiency. A pair of electrodes with a gap distance of $2 \mathrm{~mm}$ was prepared on top with silver paste or sputtered platinum. As shown in Figures $2 \mathrm{a}$ and $\mathrm{c}$, the $\mathrm{SnO}_{2}$ nanobelt network can be bent to a radius of $1 \mathrm{~mm}$ without any obvious conductance degradation. After 1000 cycles of bending to a radius of $2 \mathrm{~mm}$, the resistance of nanobelt network increased by only $110 \%$ (Figure $2 \mathrm{~d}$ ). In contrast, the electrical resistance of sputtered $\mathrm{SnO}_{2}$ thin film increased sharply by $\sim 4500 \%$ after bending to $1 \mathrm{~mm}$ (Figures $2 \mathrm{~b}$ and $\mathrm{c}$ ), and $\sim 2400 \%$ after the first 100 cycles of bending to $2 \mathrm{~mm}$ (Figure 2d). The notable mechanical durability of $\mathrm{SnO}_{2}$ nanobelt network has also been proved by monitoring the resistance change during the repeated dynamic bending test (Supplementary Figure S6 and Supplementary Video S1), which displays fully recovered electrical conductance with consecutive
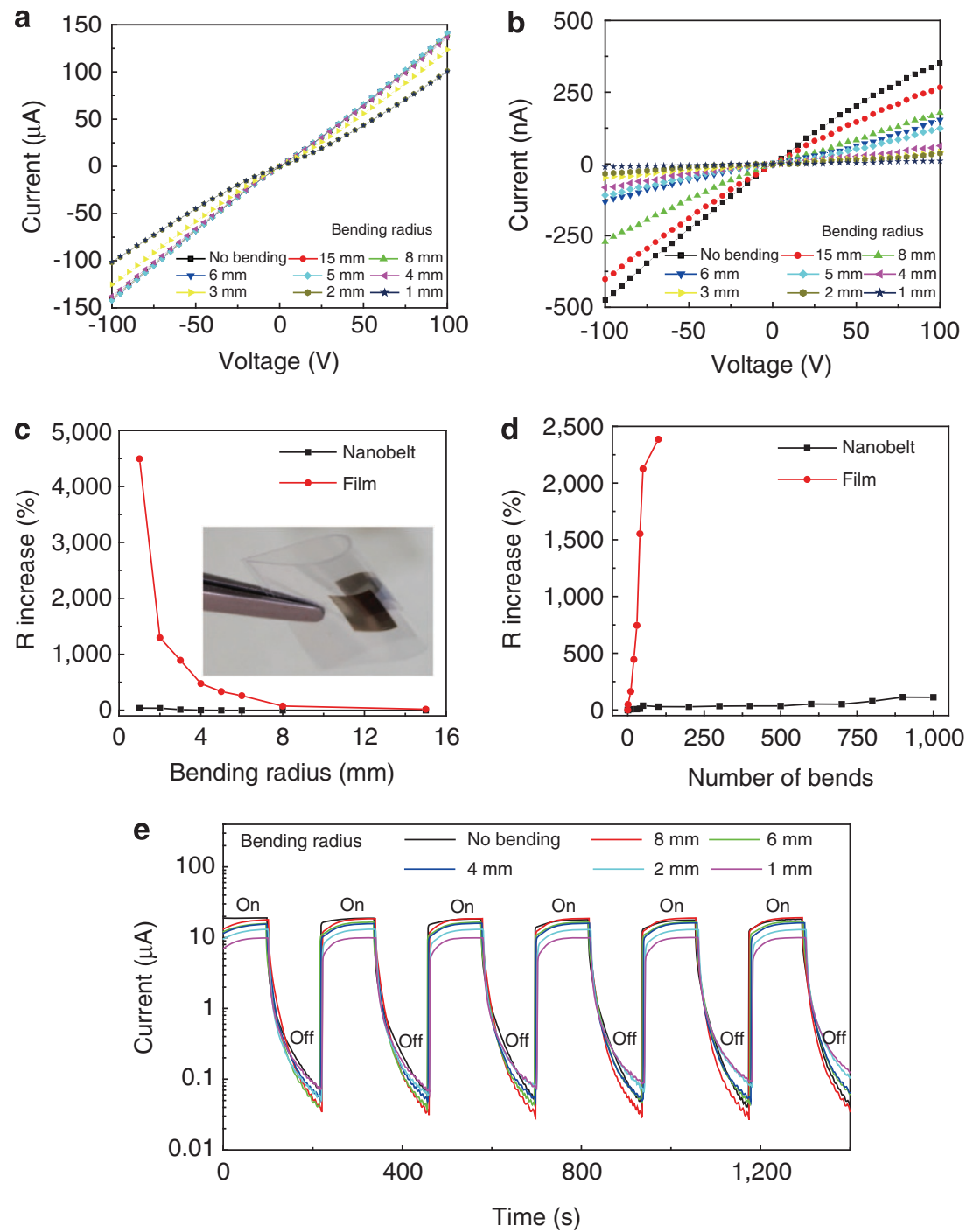

Figure 2 Mechanical flexibility and UV photoresponse of $\mathrm{SnO}_{2}$ nanobelt network. (a) The $I-V$ curves for $\mathrm{SnO}_{2}$ nanobelt network on $100-\mu m$-thick PET substrate after bending to different radii, showing no obvious conductance degradation. (b) The $I-V$ curves for $\mathrm{SnO}_{2}$ thin film on PET after bending to different radii, showing a sharp decay in electrical conductance upon bending. (c) Resistance variations of $\mathrm{SnO}_{2}$ nanobelt network and thin film on PET after bending to different radii. Inset: a digital photo of a folded $\mathrm{SnO}_{2}$ nanobelt network on PET. (d) Resistance variations of $\mathrm{SnO}_{2}$ nanobelt network and thin film on PET with different bending cycles to a radius of $2 \mathrm{~mm}$. (e) Time-dependent photoresponse of $\mathrm{SnO}_{2}$ nanobelt network under UV light illumination after bending to different radii. 
bending cycles. The small temporary increase in resistance is likely due to the increased gap distance upon bending when nanobelts undergo a nanoscale deformation to relax the applied stress. Whereas the electrical conductance of sputtered $\mathrm{SnO}_{2}$ thin film went through a rapid and irreversible decay after only the first three bending cycles (Supplementary Figure S6 and Supplementary Video S2), which may result in catastrophic failure in practical application.

Flexible optoelectronic devices based on $\mathrm{SnO}_{2}$ nanobelt network The as-assembled photodetector based on $\mathrm{SnO}_{2}$ nanobelt network exhibits high-sensitive UV photoresponse with good reversibility and reproducibility, which is believed to arise from the high surface-areato-volume ratio and well-confined 1D electron transport channel. ${ }^{27}$ Since more surface trap states may form due to the large aspect ratio, a prolonged photocarrier lifetime and thus enhanced photoconduction gain can be achieved. ${ }^{28}$ The results of bending test show that the nanobelt network can be bent to an extremely small radius $(1 \mathrm{~mm})$ and still function well despite a marginal increase in the electrical resistance (Figure 2e). Besides, the photosensitivity of $\mathrm{SnO}_{2}$ nanobelt photodetector remains above $10^{2}$ after bending to $1 \mathrm{~mm}$ (Supplementary Figure S7). For comparison, due to the greatly reduced surface area with less carrier traps, the UV photoresponse of sputtered $\mathrm{SnO}_{2}$ thin film shows a much lower sensitivity of $\sim 10$, and the electrical conduction under both dark and UV illumination decrease abruptly by $\sim 90 \%$ upon bending to $1 \mathrm{~mm}$ (Supplementary Figure S8).

\section{Mechanism of high flexibility of $\mathrm{SnO}_{2}$ nanobelt network}

The superior mechanical flexibility of nanobelt network can be further confirmed through direct observation on the microstructural change after bending test. As shown in Figure $3 \mathrm{a}, \mathrm{SnO}_{2}$ nanobelts remain a well interconnected and continuous network without detectable fracture after bending to $3 \mathrm{~mm}$. In contrast, numerous cracks on different scales appear in the sputtered $\mathrm{SnO}_{2}$ film, along with channeling and debonding (Figure 3b, and Supplementary Figure S9). This would lead to a catastrophic failure as cracks propagate through the whole film. To examine the mechanical flexibility of nanobelts under extreme deformations, we transferred them onto a piece of aluminum foil and folded it. As shown in Supplementary Figure S10, the nanobelt network preserves its integrity after unfolding. Only a few broken nanobelts are found along the crease, where the maximum strain occurred.

The extraordinary flexibility observed in the $\mathrm{SnO}_{2}$ nanobelt network originates from the unique ribbon-like nanostructure and the well-interconnected web configuration, which enable the network to maintain its structural integrity upon bending by releasing the applied stress in a nanoscale level. The nanobelt can withstand severe mechanical deformation due to its nanometer rectangular geometry. For a belt with thickness of $t$, which is bent to a radius of curvature $r$, the peak strain $\varepsilon$ can be estimated by the following equation: ${ }^{29}$

$$
\varepsilon=\frac{t}{2 r} \text {. }
$$

As for our $\mathrm{SnO}_{2}$ nanobelt with $t \sim 35 \mathrm{~nm}$, the bending radius will be $<5 \mu \mathrm{m}$ in order to create a tensile strain about $0.5 \%$, which is far beyond the requirements for most practical applications, where $r$ $\sim 1 \mathrm{~cm}$ is often sufficient. ${ }^{22}$ Besides, the contact area between nanobelts is much larger than that between nanofibers, which may further enhance the cross-junction stability of the intertwined nanobelt network.

In the meanwhile, the polycrystalline nanobelt itself shows good mechanical properties. It is known that nanocrystalline materials usually exhibit a high strength due to the Hall-Petch effect, and grainboundary sliding is proposed to be the dominant deformation mechanism at grain sizes $<50 \mathrm{~nm} .{ }^{30}$ In our experiment, the average grain size of electrospun $\mathrm{SnO}_{2}$ nanobelts is $<10 \mathrm{~nm}$ as discussed earlier. Such nanocrystalline nanobelts with ultrafine grains are supposed to tolerate severe deformation due to the enhanced strength and superplasticity caused by grain-boundary sliding.

\section{Specular transparency and optical simulation of $\mathrm{SnO}_{2}$ nanobelt network}

Optical transparency is another important performance parameter since transparent electronics are playing an increasingly important role in a wide range of areas, such as transparent displays, invisible sensors and energy conversion/storage field. ${ }^{31-33}$ As shown in Figure $4 \mathrm{a}$, over $80 \%$ optical transmittance can be obtained in the $\mathrm{SnO}_{2}$ nanobelt network. Figure $4 \mathrm{~b}$ presents an example of a highly transparent photodetector made of the $\mathrm{SnO}_{2}$ nanobelt network. The interaction between an incident light field and the nanobelt can be understood by simulation. As shown in Figures $4 \mathrm{c}$ and d, the nanobelt provides excellent light transmission with low scattering and absorption cross-sections. Particularly, the scattering cross-section is smaller than the geometrical cross-section of the nanobelt due to its sub-wavelength size effect. Figure $4 \mathrm{c}$ shows that the nanobelt has little light absorption in the visible regime. In addition, the scattering cross-section decreases at longer wavelength since the light wavelength becomes larger than the nanobelt geometrical size, which results in reduced non-resonant sub-wavelength scattering. Note that the transparency of electrospun nanofiber webs can be further improved a

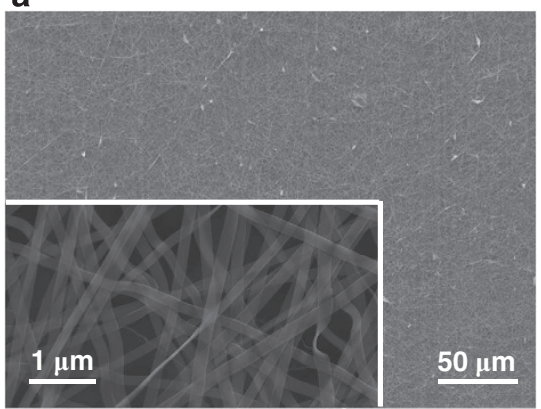

b

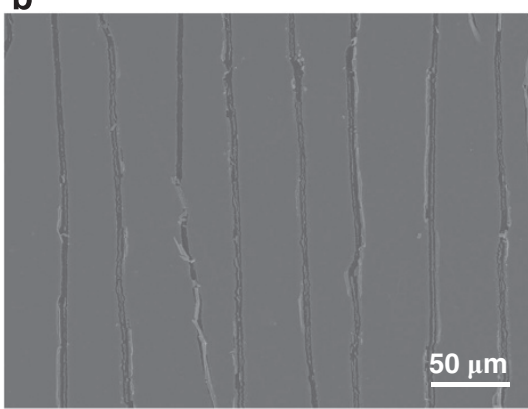

Figure 3 Microstructural evolution of $\mathrm{SnO}_{2}$ nanobelt network and sputtered thin film upon bending. (a) SEM images of a $\mathrm{SnO}_{2}$ nanobelt network after

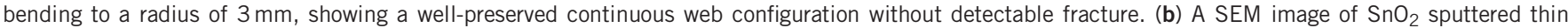
film after bending to a radius of $3 \mathrm{~mm}$, showing numerous cracks with channeling and debonding. 
by reducing the deposition time as reported in our previous studies, ${ }^{34}$ while higher mechanical flexibility can also be achieved in the nanobelt network with a smaller thickness due to the relatively low strain under the same bending radius. Herein, the nanobelt structure offers attractive advantages as flexible optoelectronics. Compared to nanofibers, the nanobelt network is supposed to have better a

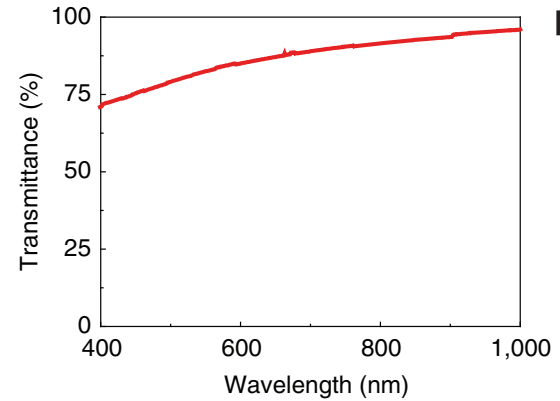

b

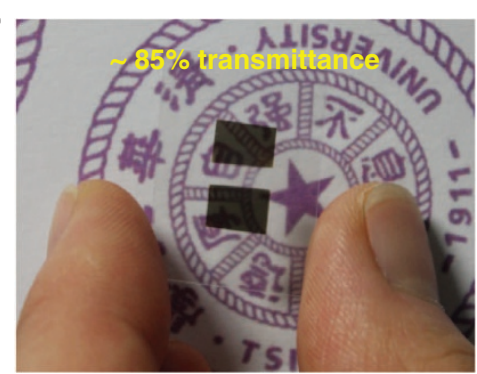

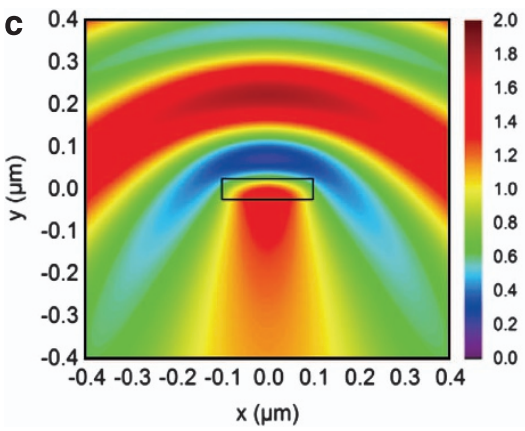

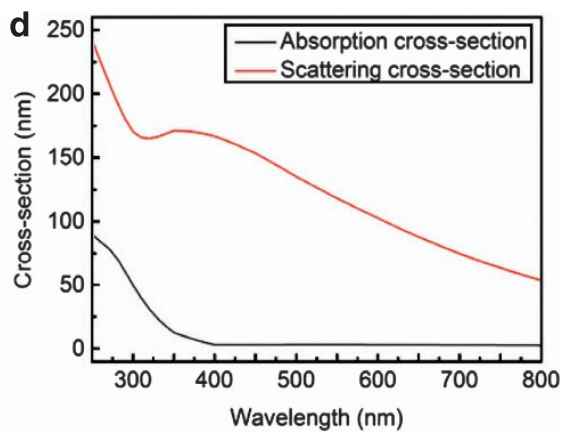

Figure 4 Spectral transmittance and optical simulation of $\mathrm{SnO}_{2}$ nanobelts. (a) Spectral transmittance of $\mathrm{SnO}_{2}$ nanobelt network on quartz (the transmittance here excludes the effect of the substrate). (b) Digital photo of a highly transparent photodetector made of $\mathrm{SnO}_{2}$ nanobelt network. (c) Electric field intensity distribution of a nanobelt for transverse electric incident light at a wavelength of $600 \mathrm{~nm}$. The nanobelt shows a smaller scattering crosssection than its geometrical size. (d) The cross-section spectra of light absorption and scattering for a single nanobelt.

a

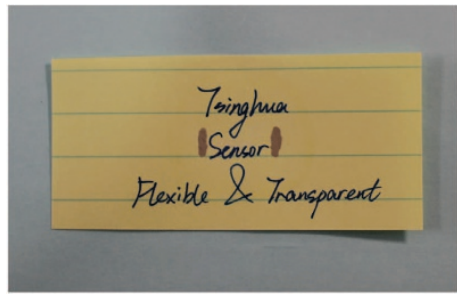

b

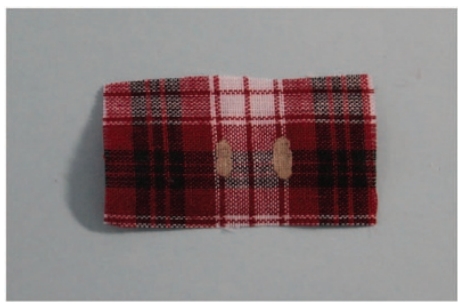

C

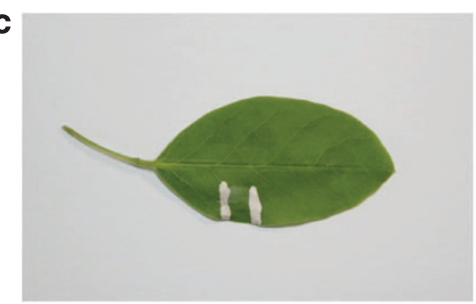

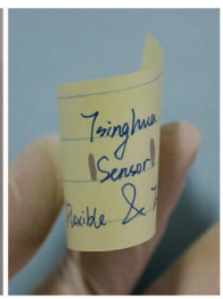
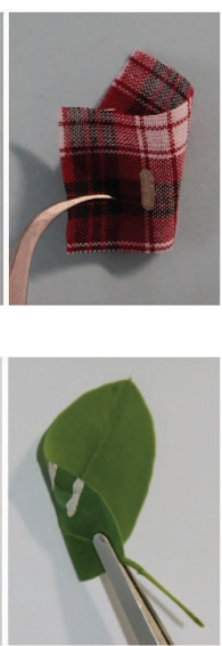
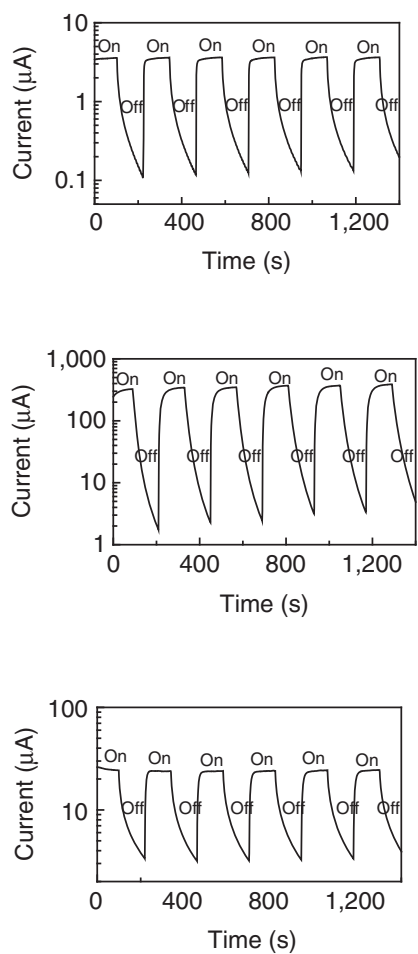

Figure 5 Flexible and transparent UV photodetectors built on various substrates. $\mathrm{SnO}_{2}$ nanobelt networks can be easily transferred to multiple substrates: (a) paper, (b) textile, (c) tree leaves and glass bottles (Supplementary Figure S11), all of which demonstrate reversible and reproducible UV photoresponse after bending to a radius of $2 \mathrm{~mm}$. 
mechanical flexibility and enhanced photoresponse owing to the tailored rectangular geometry with increased surface area, which demonstrates reliable electrical and optoelectronic performances under a bending radius down to $1 \mathrm{~mm}$. Therefore, this ceramic nanobelt network holds great potential in highly transparent and flexible electronics.

\section{Facile assembly of flexible optoelectronic devices on multiple substrates}

In combination of the outstanding mechanical flexibility and high optical transparency, we are able to construct a series of conformable and 'invisible' photodetectors. Since the nanobelt network can be manipulated in a macroscopic level as a free-standing web, we can easily transfer them onto different substrates, including paper, textiles, tree leaves and glass bottles. As shown in Figure 5 and Supplementary Figure S11, the devices formed on these soft or curved substrates can work as high-performance UV photodetectors, which demonstrate great reliability even after bending to a small radius of $2 \mathrm{~mm}$ (the photoresponse performance is influenced by the physical characteristics of different substrates).

\section{CONCLUSION}

To summarize, we have achieved high mechanical flexibility and optical transparency in inorganic semiconductors with a new nanobelt network design. Taking advantage of the closely intertwined web configuration as well as the well-tailored ribbon-like geometry, the nanobelt network exhibits remarkable mechanical flexibility and optical transparency. The free-standing nanobelt network can be easily transferred and integrated into flexible functional electronics in a cost-effective and scalable way, which enables us to construct conformable and 'invisible' UV photodetectors on various flexible and/or curved substrates. Our work presents a new strategy for designing and integrating multifunctional ceramics with high mechanical flexibility and optical transparency, which holds great potential in soft optoelectronics, such as photovoltaic systems, paperlike electronics and wearable health monitors.

\section{CONFLICT OF INTEREST}

The authors declare no conflict of interest.

\section{ACKNOWLEDGEMENTS}

This work was supported by National Basic Research of China (Grant No. 2013CB632702) and NSF of China (Grant No. 51302141).

1 Duan, X. F., Huang, Y., Cui, Y., Wang, J. F. \& Lieber, C. M. Indium phosphide nanowires as building blocks for nanoscale electronic and optoelectronic devices. Nature 409, 66-69 (2001).

2 Colvin, V. L., Schlamp, M. C. \& Alivisatos, A. P. Light-emitting-diodes made from cadmium selenide nanocrystals and a semiconducting polymer. Nature 370, 354-357 (1994).

3 Coe, S., Woo, W. K., Bawendi, M. \& Bulovic, V. Electroluminescence from single monolayers of nanocrystals in molecular organic devices. Nature 420, 800-803 (2002)

4 Feldmann, C., Justel, T., Ronda, C. R. \& Schmidt, P. J. Inorganic luminescent materials: 100 years of research and application. Adv. Funct. Mater. 13, 511-516 (2003).

5 Medintz, I. L., Clapp, A. R., Mattoussi, H., Goldman, E. R., Fisher, B. \& Mauro, J. M. Self-assembled nanoscale biosensors based on quantum dot FRET donors. Nat. Mater. 2, 630-638 (2003)

6 Chen, J., Xu, L. N., Li, W. Y. \& Gou, X. L. alpha-Fe2O3 nanotubes in gas sensor and lithium-ion battery applications. Adv. Mater. 17, 582-586 (2005).
7 Wang, X. D., Song, J. H., Liu, J. \& Wang, Z. L. Direct-current nanogenerator driven by ultrasonic waves. Science 316, 102-105 (2007).

8 Peng, H. L., Dang, W. H., Cao, J., Chen, Y. L., Wu, W., Zheng, W. S., Li, H., Shen, Z. X. \& Liu, Z. F. Topological insulator nanostructures for near-infrared transparent flexible electrodes. Nat. Chem. 4, 281-286 (2012)

9 Kim, K. S., Zhao, Y., Jang, H., Lee, S. Y., Kim, J. M., Ahn, J. H., Kim, P., Choi, J. Y. \& Hong, B. H. Large-scale pattern growth of graphene films for stretchable transparent electrodes. Nature 457, 706-710 (2009).

10 Duan, X. F., Niu, C. M., Sahi, V., Chen, J., Parce, J. W., Empedocles, S. \& Goldman, J. L. High-performance thin-film transistors using semiconductor nanowires and nanoribbons. Nature 425, 274-278 (2003)

11 Cao, Q., Kim, H. S., Pimparkar, N., Kulkarni, J. P., Wang, C. J., Shim, M., Roy, K., Alam, M. A. \& Rogers, J. A. Medium-scale carbon nanotube thin-film integrated circuits on flexible plastic substrates. Nature 454, 495-500 (2008).

12 Ridley, B. A., Nivi, B. \& Jacobson, J. M. All-inorganic field effect transistors fabricated by printing. Science 286, 746-749 (1999).

13 Talapin, D. V. \& Murray, C. B. PbSe nanocrystal solids for $n$ - and $p$-channel thin film field-effect transistors. Science 310, 86-89 (2005).

14 Kim, D. H., Lu, N. S., Ma, R., Kim, Y. S., Kim, R. H., Wang, S. D., Wu, J., Won, S. M., Tao, H., Islam, A., Yu, K. J., Kim, T. I., Chowdhury, R., Ying, M., Xu, L. Z., Li, M., Chung, H. J., Keum, H., McCormick, M., Liu, P., Zhang, Y. W., Omenetto, F. G., Huang, Y. G., Coleman, T. \& Rogers, J. A. Epidermal electronics. Science 333, 838-843 (2011).

15 Khang, D. Y., Jiang, H. Q., Huang, Y. \& Rogers, J. A. A stretchable form of single-crystal silicon for high-performance electronics on rubber substrates. Science 311, 208-212 (2006)

16 Sun, Y. G., Choi, W. M., Jiang, H. Q., Huang, Y. G. Y. \& Rogers, J. A. Controlled buckling of semiconductor nanoribbons for stretchable electronics. Nat. Nanotechnol. 1, 201-207 (2006)

17 Nomura, K., Ohta, H., Takagi, A., Kamiya, T., Hirano, M. \& Hosono, H. Room temperature fabrication of transparent flexible thin-film transistors using amorphous oxide semiconductors. Nature 432, 488-492 (2004).

18 Xu, C. N., Tamaki, J., Miura, N. \& Yamazoe, N. Grain-size effects on gas sensitivity of porous SnO2-based elements. Sensor Actuat. B-chem. 3, 147-155 (1991).

19 Gopel, W. \& Schierbaum, K. D. SnO2 sensors-current status and future-prospects. Sensor Actuat. B-chem. 26, 1-12 (1995).

20 Liu, Z. Q., Zhang, D. H., Han, S., Li, C., Tang, T., Jin, W., Liu, X. L., Lei, B. \& Zhou, C. W. Laser ablation synthesis and electron transport studies of tin oxide nanowires. Adv. Mater. 15, 1754-1757 (2003).

21 Mathur, S., Barth, S., Shen, H., Pyun, J. C. \& Werner, U. Size-dependent photoconductance in SnO2 nanowires. Small. 1, 713-717 (2005).

22 Baca, A. J., Ahn, J. H., Sun, Y. G., Meitl, M. A., Menard, E., Kim, H. S., Choi, W. M., Kim, D. H., Huang, Y. \& Rogers, J. A. Semiconductor wires and ribbons for highperformance flexible electronics.. Angew. Chem. Int. Edit. 47, 5524-5542 (2008).

23 Li, D. \& Xia, Y. N. Electrospinning of nanofibers: Reinventing the wheel? Adv. Mater $16,1151-1170$ (2004)

24 Greine, A. \& Wendorff, J. H. Electrospinning: A fascinating method for the preparation of ultrathin fibres. Angew. Chem. Int. Edit. 46, 5670-5703 (2007).

25 Fong, H., Liu, W. D., Wang, C. S. \& Vaia, R. A. Generation of electrospun fibers of nylon 6 and nylon 6-montmorillonite nanocomposite. Polymer. (Guildf). 43, 775-780 (2002).

26 Krishnappa, R. V. N., Desai, K. \& Sung, C. M. Morphological study of electrospun polycarbonates as a function of the solvent and processing voltage. J. Mater. Sci. $\mathbf{3 8}$ 2357-2365 (2003).

27 Peng, L. F., Hu, L. \& Fang, X. S. Low-dimensional nanostructure ultraviolet photodetectors. Adv. Mater. 25, 5321-5328 (2013).

28 Liu, S., Ye, J. F., Cao, Y., Shen, Q., Liu, Z. F., Qi, L. M. \& Guo, X. F. Tunable hybrid photodetectors with superhigh responsivity. Small. 5, 2371-2376 (2009).

29 Suo, Z., Ma, E. Y., Gleskova, H. \& Wagner, S. Mechanics of rollable and foldable filmon-foil electronics. Appl. Phys. Lett. 74, 1177-1179 (1999).

30 Meyers, M. A., Mishra, A. \& Benson, D. J. Mechanical properties of nanocrystalline materials. Prog. Mater. Sci. 51, 427-556 (2006).

31 Gorrn, P., Sander, M., Meyer, J., Kroger, M., Becker, E., Johannes, H. H., Kowalsky, W. \& Riedl, T. Towards see-through displays: Fully transparent thin-film transistors driving transparent organic light-emitting diodes. Adv. Mater. 18, 738-741 (2006).

32 Yang, Y., Jeong, S., Hu, L. B., Wu, H., Lee, S. W. \& Cui, Y. Transparent lithium-ion batteries. Proc. Natl Acad. Sci. USA 108, 13013-13018 (2011).

33 Wang, A., Babcock, J. R., Edleman, N. L., Metz, A. W., Lane, M. A., Asahi, R., Dravid, V. P., Kannewurf, C. R., Freeman, A. J. \& Marks, T. J. Indium-cadmium-oxide films having exceptional electrical conductivity and optical transparency: clues for optimizing transparent conductors. Proc. Natl Acad. Sci. USA 98, 7113-7116 (2001).

$34 \mathrm{Wu}, \mathrm{H}$., Hu, L. B., Rowell, M. W., Kong, D. S., Cha, J. J., McDonough, J. R., Zhu, J., Yang, Y. A., McGehee, M. D. \& Cui, Y. Electrospun metal nanofiber webs as highperformance transparent electrode. Nano. Lett. 10, 4242-4248 (2010).

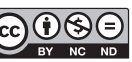

This work is licensed under a Creative Commons Attribution-NonCommercial-NoDerivs 3.0 Unported License. To view a copy of this license, visit http://creativecommons. org/licenses/by-nc-nd/3.0/

Supplementary Information accompanies the paper on the NPG Asia Materials website (http://www.nature.com/am) 